trop de semence et de laisser les récipients à la température de $40^{\circ}$; dans ces conditions la coagulation se manifeste dans l'espace de trois à quatre heures; les Yo-ourts préparés ainsi peuvent être conservés sans aigrir et sans perdre leur saveur, en les laissant mème longtemps à l'étude de $37^{\circ}$.

Si on ajoute à ceux-ci le bacille homogène, le Yo-ourt devient un peu plus aigre (nous avons indiqué les inconvénients de la présence des levures).

Actuellement nous avons les cultures pures des bactéries (agents du Yo-ourt) et les conditions nécessaires étant déterminées, la préparation d'un Yo-ourt délicieux et hygiénique a été ainsi assurée.

\title{
OBSERVATIONS SUR UNE JEUNE GUENON NÉE EN CAPTIVITÉ \\ ET ÉLEVÉE AU BIBERON DĖS SA NAISSANCE
}

\author{
Par M. DIDIER
}

\author{
Docteur-vétérinaire,
Directeur du Jardin zoologique du Pare de la Tête-d'Or, à Lyon.
}

"Ginette ", la petite guenon qui fait l'objet de cette observation, est née, dans la matinée du 7 juin 192' Pare de la Tête-d'Or, à Lyon.

Elle est le produit d'un macaque commun des Indes (macacus sinicus), âgé de sept ans environ, et d'une guenon de Barbarie ou Magot (macacus inuus), âgée de trois ans environ. Ces animaux étaient. placés dans la même cage depuis janvier 1923.

Au moment de la mise-bas, la mère meurt subitement d'une embolie, alors què son petit, bien vivant, est encore reliè à elle par le cordon ombilical, qu'il faut ligaturer et couper pour séparer du cadavre le nouveau-né, destiné à être élevé artificiellement.

Dès sa naissance, on le revêt d'une petite chemise en jersey de laine, qu'on recouvre, pour la nuit, d'un autre vêtement plus épais, en laine tricotée. Un panier d'osier fermé, garni de couvertures et d'une bouillotte, lui sert de berceau pendant les six premiers jours (la bouillotte n'est employée à partir du quatrième jour que pour la nuit seulement). Ensuite, le panier est remplacé par une corbeille de bureau, dans laquelle Ie jeune sujet fera bientôt ses débuts d'équilibriste.

Alimentation. - L'alimentation consiste, le premier jour, en eau sucrée, pour faciliter l'évacuation du méconium qui se fait normalement. Le deuxième jour, on donne un quart de litre de lait de vache coupé d'eau sucrée (moitié lait, moitié eau sucrée), jusqu'au 20 juin, époque à laquelle on commence l'emploi de la farine lactée (une cuillerée à café environ) pour la nuit, à seule fin de rendre 
l'animal moins exigeant et de lui laisser plus de sommeil. Les tétées diurnes et nocturnes, qui se faisaient chaque deux ou trois heures, ne se renouvellent plus que quatre à cinq fois par vingt-quatre heures à partir du $1^{\mathrm{er}}$ août. Elles sont de 250 gr. de lait de vache bouilli, tiède et sucré. Le biberon est tonjours accueilli avec la plus grande satisfaction (fig. 1).

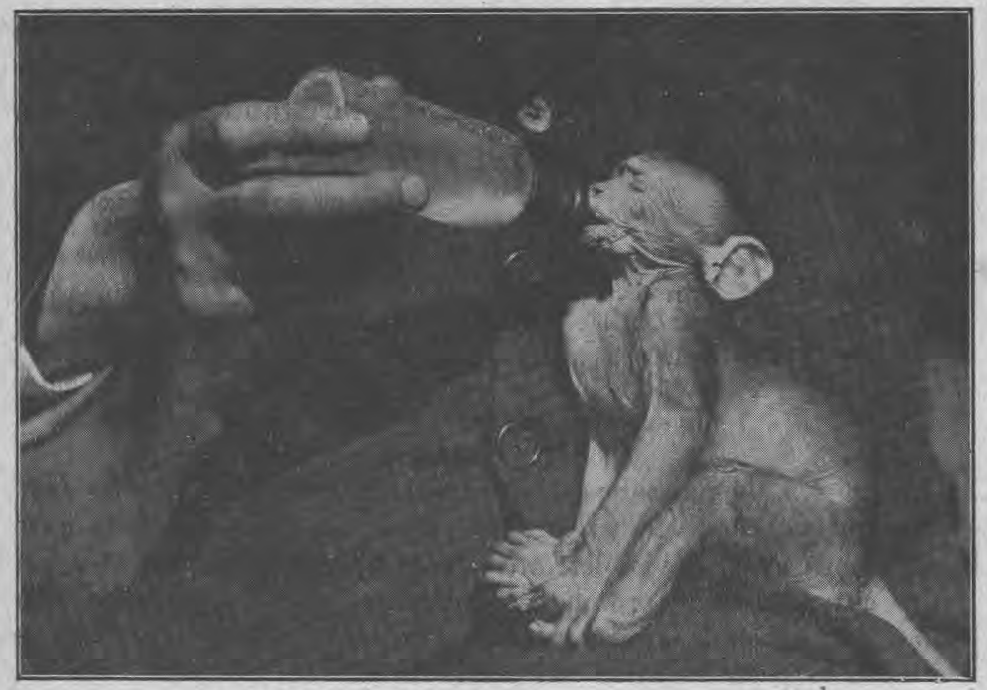

FIG. 1. - Jeune guenon alimentée au biberon.

Maladroitement d'abord, la petite guenon commence à porter à sa bouche des fruits qu'elle suce, et dont elle rejette vite les débris ; puis, au bout de quelques jours, les progrès sont très sensibles. Elle mange avec beaucoup de grâce et d'adresse, des fruits à pulpe, très mûrs et très sucrés, qu'elle sait choisir (prunes principalement), des légumes aqueux (tomates surtout), grignote croûtes de pain, gâteaux, chocolat même, et tout ce qui peut tomber sous sa... main. Les préférences s'accentuent de jour en jour, la gourmandise se manifeste et l'esprit d'imitation apparaît, car la jeune Ginette veut aussi boire au verre, comme les gens de son entourage. Inutile d'ajouter qu'elle n'abandonne pas pour cela le biberon, qui lui est toujours nécessaire, surtout à l'heure du sommeil. Cependant, on a pu parfaitement la serrer à l'âge de quatre mois et demi.

Tarlle. Poids. - En raison de l'alimentation choisie et des soins attentifs dont on l'entoure, le sujet se développe visiblement.

Le poids, qui est au jour de la naissance de 585 gr., augmente de la facon suivante : 


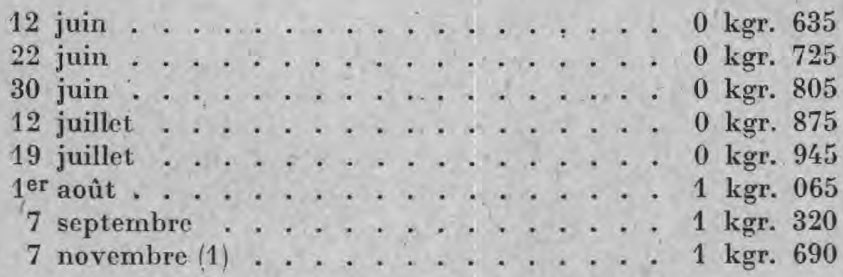

Le périmètre thoracique passe de $0 \mathrm{~m} .19$ ( 30 juin) à $0 \mathrm{~m}, 205$ (19 juillet), et de $0 \mathrm{~m} .22$ (1 ${ }^{\mathrm{er}}$ août) à $0 \mathrm{~m} .26$ ( 7 novembre).

La taille est très difficile à prendre, vu l'irritabilité et la vivacité de l'animal, qui se fâche et se replie sur lui-même.

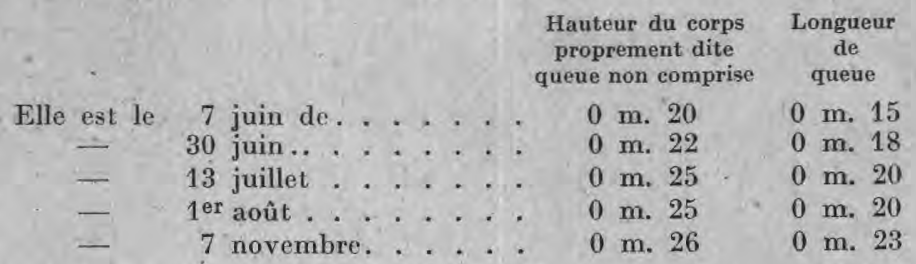

Dentition. - A la naissance, on constate la présence de deux incisives supérieures et de quatre incisises inférieures. Les deux incisives externes inférieures sont à peine visibles, et les supérieures commencent à pointer les jours suívants. Les premières molaires percent la gencive vers le 19 juillet. Peu après, le sujet possède à chaque mâchoire : quatre incisipes, deux canines et deux molaires très apparentes.

Actuellement (14 décembre 1924) : quatre molaires.

Locomotion. - Vers le 20 juillet, la jeune "Ginette ", du fond de sa corbeille, essaie, en grimpant, d'en atteindre les bords, et arrive très vite à en sortir seule. Puis elle commence à marcher gauchement, sur ses quatre pattes, titube, tombe et s'exerce sans relâche. Elle devient bientôt très agile, et fait des bonds qui paraissent chaque jour plus audacieux.

Comme elle se montre alors insupportable en liberté continue, qu'elle s'attaque notamment aux objets fragiles et brillants (cristaux, robinets à gaz, épingles, etc.), au papier de tenture, qu'elle déchire par lambeaux, on se voit obligé de l'enfermer dans une cage d'oiselier, dont elle sait bientôt, du reste, ouvrir la porte close par un simple erochet.

Aspect général. - Voici l'aspect général de la jeune guenon pendant les premiers jours de sa vie :

Face : ridée, couleur chair.

Yeux : noirs, vifs, très expressifs.

Oreilles : arrondies, plates, minces, présentant dans leur partie supérieure un repli horizontal très marqué:

Corps : plutôt grêle.

Membres : longs, principalement les jambes.

Pieds et mains : avec pouce opposable et ongles parfaitement formés.

(1) C'est-à-dire à l'âge de cinq mois. 
Les pieds ont la même structure que les mains, mais sònt de dimensions beaucoup plus grandes (quadrumanes).

Queue : pas prenante, mais semble être le balancier nécessaire aux rétablissements agiles de la petite bête.

Pelage : brun-jaunâtre, clairsemé au cou, sur la poitrine, à la face interne des membres et sur l'abdomen, dont la peau est bleuâtre. Les poils de İa tête sont noirs et descendent bas sur le front, où ils forment une ligne horizontale très nette, coupée par la raie du milieu du crâne. Au sommet, on remarque un épi particulier.

Vers le milieu de juillet, le pelage s'allonge sur la poitrine. Il épaissit par tout le corps, qui prend une jolie teinte jaunâtre. Les parties glabres du début de la vie sont presque entièrement recouvertes de poils plus ou moins touffus.

En résumé, cette petite bête est très vigoureuse et ne semble point souffrir de n'avoir pas été allaitée par sa mère. Elle s'alimente avec régularité ; toutes les fonctions sont normales. Elle dort toute la nuit, mais les sommeils de la journée sont rares et courts.

Quoique d'un caractère irascible et souvent prête à mordre, elle est très affectueuse pour les personnes qui la soignent le plus souvent et pour celles qui lui sont sympathiques. Très sensible aux corrections, dont la menace suffit quelquefois pour la remettre au calme, elle vient se blottír dans les bras, avec confiance, comme le ferait un enfant pour se faire caresser et pardonner.

Ses jeux de physionomie sont variés et révèlent fort bien le sentiment éprouvé. Pour exprimer sa joie, sa tendresse, elle se déplisse la face d'une manière tout à fait particulière, fait entendre un clappement caractéristique et grognonne à la facon des macaques adultes. Pour manifester son mécontentement, son impatience - ou sa peur, en cas de solitude - elle jette des cris déchirants, qui ressemblent parfois à des piaillements ou à des sifflements d'oiseaux. Lorsque ces accès de colère lui prennent en cage, elle se calme très rapidement dès qu'elle voit'apparaître quelqu'un de connaissance, et se couvre drôlement la tête d'un coin de sa couverture ou du chiffon qui lui sert de jouet, laissant voir cependant le visage encore grimaçant.

A tout instant, mais surtout à l'heure du sommeil ou de la faim, elle saisit avec sa main droite son gros orteil, qu'elle tette inlassablement jusqu'à réalisation de son désir.

Nous eroyons devoir ajouter enfin que cette jolie petite femelle était vouée à une mort certaine, si nous n'avions décidé, immédiatement après la mise-bas, de la faire élever artificiellement à la maison, n'ayant pas cru pouvoir demander aux employés du jardin zoologique de s'astreindre à un élevage aussi délicat.

Actuellement (14 décembre 1924), ce petit singe se porte admirablement, mais ne pourra vraisemblablement prendre place dans les collections du Jardin zoologique qu'après la saison froide, quand il sera possible de lui faire supporter, sans risque, le régime habituel des singes adultes et acclimatés au Parc de la Tète-d'Or. 
Cette observation m'a paru intéressante à signaler, étant donné la rareté des cas de reproduction des singes en captivité, sous le climat lyonnais et, surtout, quand le sujet est le produit de l'accouplement d'un macaque commun des Indes (macacus sinicus $\sigma^{*}$ ) avec une guenon de Barbarie ou Magot (macacus inuus 우).

Nous ferons remarquer, en outre, les difficultés qu'il y a à élever, artificiellement, au biberon et avec un plein succès, dès le premier jour de sa naissance, une jeune guenon.

Pour arriver à un bon résultat, il faut lui prodiguer, surtout au début de son élevage, des soins entendus, de tous les instants (la nuit comme le jour), pour ainsi dire maternels et, aussi, une patience à toute épreuve.

\title{
LE PROCÈS DE LA MATIÉRE GRASSE DU LAIT
}

\author{
par Ch. PORCHER
}

- SUITF -

\section{La matière grasse et l'extrait dégraissé brut. L'extrait} dégraissé rectifié. - Quand on considère les oscillations du taux de la matière grasse dans un lait individuel, - et nous savons qu'elles peuvent être considérables, - nous voyons en même temps que le taux de l'extrait dégraissé varie en sens inverse de celui de la graisse, à tel point que des laits extrêmement gras peuvent donner l'apparence d'être mouillés sur le simple ou du chiffre de l'extrait dégraissé au litre.

L'étude analytique des divers prélèvements d'une même traite met d'ailleurs très bien cette notion en évidence, et e'est parce qu'il y avait lieu de penser que la qualité chimique de l'extrait dégraissé ne devait pas varier depuis le commencement jusqu'à la fin de la traite, alors qu'elle subissait, en apparence, des modifications profondes, que j'ai été amené à proposer un calcul de rectification dont il va être question maintenant (1).

Si nous nous reportons à la fig. 25 qui traduit les observations de Carlloux, et que nous en distrayons l'observation $\mathbf{n}^{0} 4$, en y ajoutant les indications analytiques relatives au lactose, à la caséine et aux cendres, nous relevons le tableau suivant qui nous permet de construire la fig. 71 .

\begin{tabular}{|c|c|c|c|c|c|c|c|}
\hline $\begin{array}{l}\text { Imporn } \\
\text { es,fonctipn }\end{array}$ & ents & $\begin{array}{l}\text { Matière } \\
\text { grasse }\end{array}$ & Lactose & Caséine & Cendres & $\begin{array}{c}\text { Extrait } \\
\text { sec }\end{array}$ & $\begin{array}{l}\text { Extrait } \\
\text { dégraissé }\end{array}$ \\
\hline $1.000 \mathrm{ec}$ & & 12,32 & 45,24 & 35,25 & 7,80 & 104,37 & 92,05 \\
\hline $1.000-$ & & 29 , & 44 & 34,1 & & 1 & \\
\hline 1.000 & . & 39,48 & 43, & 32,06 & 7, & 127,32 & 87,84 \\
\hline 1.000 & 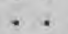 & 50,06 & 42,32 & 31,81 & 7,01 & 135,74 & 85,68 \\
\hline 400 & & 91,17 & 40,65 & 30,20 & 7,08 & 137,36 & 82,19 \\
\hline
\end{tabular}

(1) Ch. Porcher, Influence du taux de la matière grasse sur celuí de l'extrait dégraissé dans le lait (Annales des Falsifications, p. 385, nov.-déc. 1915). 